\title{
JOB CATEGORY DIFFERENCES IN THE PREVALENCE AND ASSOCIATED FACTORS OF INSOMNIA IN STEEL WORKERS IN CHINA
}

\author{
XIAOMING LI, SHIYUE CUI, JIANHUI WU, LIHUA WANG, and JUXIANG YUAN \\ North China University of Science and Technology, Tangshan, China \\ School of Public Health
}

\begin{abstract}
Objectives: This study aimed to investigate the prevalence of insomnia and risk factors among different job categories of steel workers in China, in order to improve their quality of occupational life. Material and Methods: A cross-sectional face-to-face survey was conducted which involved 5834 steel workers from a large enterprise located in northern China, including front-line, maintenance and inspection, and other auxiliary workers. The Athens Insomnia Scale and the Job Content Questionnaire were used to assess the status of insomnia and job stress/social support, respectively. Multivariable logistic regression was used to identify factors influencing insomnia. Results: The overall prevalence of insomnia was determined at $42.0 \%$ (95\% confidence interval: 40.7\%-43.2\%). For front-line, maintenance and inspection, and other auxiliary workers, the prevalence was 42.3\%, 39.8\%, and 47.9\% (p = 0.001), respectively. The participants with high stress and low support, and those who had experienced $\geq 2$ major life events in the past 12 months, compared to those with low stress and high support, and those without major events, displayed an increased risk of insomnia among all 3 job categories (the adjusted odds ratio ranged 1.56-2.38 and 1.30-1.75, respectively). The educational level, shift work, alcohol consumption, and present illness were identified as influencing factors of insomnia for 1 or 2 job categories. Conclusions: The prevalence of insomnia was the highest in the group of other auxiliary steel workers among the 3 job categories of steel workers under consideration. While the influencing factors of insomnia differed among the groups, job stress and major life events were common risk factors of insomnia among the 3 categories of steel workers. Int J Occup Med Environ Health. 2020;33(2):215-33
\end{abstract}

Key words:

insomnia, shift work, job stress, life events, steel workers, job category

\section{INTRODUCTION}

At present, insomnia is one of the most common and widely recognized public health problems all over the world. In previous studies [1-4], the prevalence of insomnia ranged $7.1-79.8 \%$ in general populations in the world, e.g., $7.1 \%$ in Norway [2], 22.1\% in the USA [1], 11.9\% in Hong Kong of China [3], and 79.8\% in Brazil [4]. In China, studies have shown that the prevalence of insomnia ranges $12.7-55.7 \%$ in different populations [5-8]. Although the prevalence of insomnia varies considerably in terms of criteria and symp- toms, the prevalence above $30 \%$ for the global general population is commonly accepted. Meanwhile, several studies have reported that the prevalence of insomnia varies by job type [9-11]. For example, the prevalence of insomnia was determined at $23.2 \%$ among workers in the USA [9], $31 \%$ among manufacturing workers in South Korea [10], 23.2\% among male public service workers in Japan [11], and $16.8 \%$ among financial workers in France [12].

Insomnia is said to increase the risk of numerous physical and mental diseases, including heart disease, depression,

Funding: this research was supported by the Ministry of Science and Technology of the People's Republic of China (project No. 2016YFC0900605 entitled "National Key R\&D Program of China," project manager: Juxiang Yuan, Ph.D.).

Received: July 16, 2019. Accepted: December 30, 2019.

Corresponding author: Juxiang Yuan, North China University of Science and Technology, School of Public Health, 21 Bohai Road, Caofeidian Xincheng, Tangshan, Hebei, China (e-mail: yuanjx@ncst.edu.cn). 
hypertension, anxiety, stroke, dyslipidemia, atrial fibrillation, obesity, migraine [13], and metabolic syndrome [14]. Furthermore, insomnia is associated with poor work performance, absenteeism, accidents at work $[9,10]$, reduced work productivity, increased workplace costs, and a declined quality of working life [15]. According to an American study, the direct economic costs attributed to insomnia amounted to USD 13.9 billion in 1995 and were projected to increase year-by-year [16].

Such a high prevalence and a multitude of adverse effects suggest that epidemiological studies of workplace insomnia are extremely necessary. There are a lot of occupational hazards that can increase the risk of insomnia, such as hazardous gas, noise, hot environment, strenuous physical work, job stress, night work, etc. [17-19]. However, the prevalence of insomnia and its associated factors are rarely known in steel workers, especially in China.

Several socio-demographic, psychological, lifestyle- and behavior-related, as well as occupational factors are associated with insomnia, including anxiety, physical health, religious beliefs [5], sex [6], alcohol consumption [20], a lack of steady income, less frequent social contacts, reduced social capital, living alone [7], shift work [21], occupational stress [8], etc. However, little is known about whether the prevalence of insomnia and its associated factors are different across job categories of steel workers. This hinders the ability of decision-makers to introduce relevant policies that may protect workers' health and reduce workplace costs. Therefore, the present study aimed to investigate the prevalence of insomnia and its associated factors among different job categories of steel workers in China.

\section{MATERIAL AND METHODS}

\section{Participants}

The participants were recruited from the Tangsteel company, a large steel enterprise in the Hebei Province in northern China. All 8646 steel workers who participated in the annual occupational health examination in 2017 had been selected as potential research subjects, from whom 5896 workers eventually participated and completed the survey voluntarily. There were 48 subjects whose demographic information was missing, and 14 subjects who lacked more than a third of the items in the insomnia scale; these 62 subjects were, therefore, rejected. Thus, 5834 participants were included in the final analysis. Data collection was performed in March-July 2017. All the subjects had given their informed consent for inclusion before they participated in the study. The study was conducted in accordance with the Declaration of Helsinki, and the protocol was approved by the Ethics Committee of the North China University of Science and Technology (No. 16040).

The mean age of the subjects was $42.9( \pm 8.6)$ years, and $91.3 \%$ were male. As regards the educational level, junior high school education was reported by $23.2 \%$, high school education by $51.9 \%$, and college or university education by $24.9 \%$ of the participants. As regards the marital status, $92.4 \%$ of the participants were married, $4.7 \%$ unmarried, and $2.9 \%$ separated or divorced.

\section{Measures}

\section{Questionnaire measurements}

All the participants were interviewed face-to-face by members of the research team following a structured questionnaire. The questionnaire consisted of 5 parts:

- socio-demographic characteristics (sex, age, educational level, marital status, household income, household living area, etc.),

- living and behavioral habits (smoking, alcohol consumption, tea consumption, physical activity, etc.),

- job characteristics (shift work, employment relationship, job category, job stress, etc.),

- personal characteristics (height and weight, life events, present illness),

- an assessment of insomnia. 


\section{Definition of smoking}

Current smokers were those who had regularly consumed $\geq 1$ cigarette/day for at least the past 12 months. Some participants who had quit cigarettes within the past 12 months were also assigned as current smokers. Ever smokers were those who had quit cigarettes earlier than 12 months before. Never smokers were those who had never smoked in their lifetime.

\section{Alcohol consumption status}

Never drinkers were those who had not consumed alcohol in their lifetime. Ever drinkers were those who had once consumed alcohol, but had not done so for the past 6 months. Alcohol consumption was calculated according to the following formula:

\author{
Alcohol consumption $(\mathrm{g} /$ day $)=$ \\ [liquor $(\mathrm{kg} /$ month $) \times 1000 \times 0.45+$ \\ beer (bottle/month) $\times 640 \times 0.036+$ \\ wine/fruit wine $(\mathrm{kg} /$ month $) \times 1000 \times 0.0096] / 30$
}

\section{Tea drinking}

Tea drinkers were those who drank $\geq 1$ cup of Chinese black tea, green tea, or jasmine tea per week, continuously for at least 6 months.

\section{Physical activity}

The authors assessed physical activity using the Chinese version of the International Physical Activity Questionnaire (IPAQ) [22,23]. The Chinese version of IPAQ had been previously identified as having an acceptable reliability and validity. The participants were asked to supply information on their physical activity during the previous 7 days at work, at home, and any recreational or sport, and leisuretime activities. Activities that the individual performed for at least $10 \mathrm{~min}$ at a time were collected along with the total duration per day, the number of days, and the intensity (vigorous, moderate, or low) of each activity.
For each individual, the authors assessed their physical activity according to the guidelines for data processing and analysis of IPAQ. The recorded activities of the participants were converted into metabolic equivalent task (MET) [min/week]. The individuals with MET values $<600$ were classified as having a low level of physical activity, those with MET values 600-3000 as having a moderate level of physical activity, and those with MET values $>3000$ as having a high level of physical activity.

\section{Employment relationship}

The individuals who had signed long-term service (until retirement) contracts with the steel company were classified as contract workers; those who had not signed long-term service contracts, but only short-term service contracts or temporary service contracts, were classified as other forms; some people who were assigned to work by the labor dispatch agency, but had not signed any service contracts with the steel company, were also classified as other forms.

\section{Shift work}

A majority of the participants worked according to a 4-team/3-shift schedule, so this shift type was analyzed as a separate group. In this group, according to the International Labour Organization (ILO) [24], the day-shift worker is defined as a person working day shift at 8:00-16:00, evening shift at 16:00-24:00, or night shift at 24:00-8:00 of the following day. Some of the participants worked according to a 3-team/2-shift, 5-team/3-shift, or 4-team/ 2-shift schedule, and they were divided into other shift type groups. The rest of the participants who constantly worked according to a day-shift schedule, without night shifts, were assigned to the day-shift group.

\section{Job stress}

The Job Content Questionnaire (JCQ) developed by Karasek [25] was employed for evaluating job stress in this study. The reliability and validity of the Chinese version of 
JCQ had been previously proven [26]. There are 22 items included in JCQ which can be divided into 3 scales: job demands (5 questions), job control or job decision latitude (9 questions), and social support (8 questions). Every item was scored on a Likert scale from 1 (rarely or never) to 4 (constantly or always). Job demands, job control, and social support were separately calculated by adding up the scores for each item.

The authors evaluated stress using the demand-control ratio $(\mathrm{D} / \mathrm{C}$ ratio), calculated in the following way:

(the scores of demand)/(the scores of control) $\times$ (correction factor $(9 / 5)$ )

A D/C ratio of $>1$ was considered as high stress; otherwise, it was considered as low stress. The social support dimension was divided into low and high groups according to the specific distribution median. Job stress was divided into 4 groups by combining the $\mathrm{D} / \mathrm{C}$ ratio and social support groups: low stress and high support, low stress and low support, high stress and high support, and high stress and low support. According to the Demand, Control and Support (DCS) model, also developed by Karasek [25], the high stress and low support group is expected to experience the highest levels of stress while the low stress and high support group the lowest levels of stress. The other 2 groups are expected to experience moderate levels of stress.

\section{Life events}

The authors recorded major life events during the past 12 months that were self-reported by the participants. The list of these life events included divorces or separations, conflicts, deaths, serious illnesses, marriages and childbirths, which occurred in any member of their family. The participants were given a score of 0 for no such events, 1 for 1 such event, 2 for 2 such events, and so on.
Present health status

The authors requested the participants to self-report all information regarding their current illnesses which had been diagnosed by physicians. The list of current illnesses included respiratory diseases such as chronic bronchitis, asthma, emphysema, phthisis, chronic rhinitis, and non-respiratory (other) diseases such as hypertension, cardiovascular disease, chronic gastritis, rheumatoid arthritis, diabetes, gout, thyroid disorders, liver disease, and alcoholism.

\section{Assessment of insomnia}

The Athens Insomnia Scale (AIS), which is widely used for assessing insomnia in many countries [27,28], was employed as the insomnia assessment tool in this study. The scale consisted of 8 items, each of which was scored on a 4-point Likert scale from 0 (no problem at all) to 3 (a very serious problem), with the total score ranging $0-24$. A score of $\geq 6$, with 6 being the widely accepted cut-off value for insomnia, classified the participant into the insomnia group; other participants were classified to the non-insomnia group.

\section{Job categories}

According to work content and workshops, all the participants were divided into the following 3 groups: front-line workers, maintenance and inspection workers, and other auxiliary workers. Front-line workers included the workers who engaged in iron-making, steel-making, hot steel rolling, and cold steel rolling. Maintenance and inspection workers were workers who mainly engaged in the monitoring and maintenance of steel-making instruments and equipment, and the testing of steel products. Other auxiliary workers included workers who engaged in steel deep processing, steel products logistics, and furnace material making.

\section{Statistical analysis}

Data were analyzed using SPSS 20.0 for Windows (IBM, Armonk, NY, USA). The socio-demographic characteris- 
tics, living and behavioral habits, job characteristics, personal characteristics, and insomnia were described as percentage values of categorical variables. The comparison of the characteristics and percentage of insomnia across different job categories were analyzed using $\chi^{2}$ tests. A binary logistic regression analysis was used to analyze the association between essential characteristics and insomnia across different job categories, and crude odd ratios (ORs) were calculated. Adjusted odds ratios (aORs) for insomnia and the $95 \%$ confidence intervals (95\% CIs) of each factor were separately calculated, while other factors were adjusted. In this study, $\mathrm{p}<0.05$ was established as the significance level.

\section{RESULTS}

Among the 5834 participants, the groups of front-line workers, maintenance and inspection workers, and other auxiliary workers comprised 2848, 2299 and 687 participants, respectively. The comparison of essential characteristics across the groups representing different job categories are shown in Table 1. The distribution of sex, age, educational level, marital status, household per capita income, household per capita living area, body mass index (BMI), smoking status, alcohol consumption, tea drinking, physical activity, employment relationship, shift work, job stress, life events, and present illness were significantly different among the 3 job categories (alcohol drinking factors, $\mathrm{p}=0.001$; life events factors, $\mathrm{p}=0.015$; other factors, $\mathrm{p}<0.001)$.

The prevalence of insomnia across the groups representing the 3 job categories with different essential characteristics is compared in Table 2. The prevalence of insomnia was $42.0 \%$ in the total steel workers, and was the highest among other auxiliary workers (47.9\% vs. $42.3 \%$ for frontline workers, vs. $39.8 \%$ for maintenance and inspection workers, $p<0.001$ ). The prevalence of insomnia among other auxiliary workers was higher than in other groups of steel workers representing different essential charac- teristics. These essential characteristics included sex, age of 30-49 years and 40-49 years, a high level of education (high school, college/university and above), being married, household per capita income, household per capita living area of $\geq 20 \mathrm{~m}^{2}$, a BMI group of 18.5-24.9, never smoking or smoking 1-20 cigarettes/day, never drinking alcohol, tea drinking, light or heavy physical activity, contract workers, a 3-shift/4-team work schedule, high job stress, and experiencing life events in the past 12 months ( $p<0.05)$.

The associations were observed between each essential characteristic and insomnia using logistic regression analyses, as shown in Table 3. Compared to low stress and high support workers, low stress and low support workers (front-line workers: aOR $=1.50,95 \% \mathrm{CI}$ : 1.15-1.95; maintenance and inspection workers: $\mathrm{aOR}=1.37,95 \% \mathrm{CI}$ : 1.02-1.84; other auxiliary workers: $\mathrm{aOR}=2.46,95 \% \mathrm{CI}$ : 1.33-4.54), and high stress and low support workers (front-line workers: $\mathrm{aOR}=2.28,95 \% \mathrm{CI}$ : $1.85-2.81$; maintenance and inspection workers: $\mathrm{aOR}=1.56,95 \% \mathrm{CI}$ : 1.23-1.98; other auxiliary workers: $\mathrm{aOR}=2.38,95 \% \mathrm{CI}$ : 1.52-3.73) have a higher risk of insomnia among the 3 job categories.

Compared to the workers who had not experienced any major life events in the past 12 months, the workers who had experienced $\geq 2$ such life events in the past 12 months had a higher risk of insomnia among the 3 job categories (front-line workers: $\mathrm{aOR}=1.72,95 \% \mathrm{CI}$ : 1.40-2.11; maintenance and inspection workers: $\mathrm{aOR}=1.30,95 \% \mathrm{CI}$ : 1.02-1.65; other auxiliary workers: $\mathrm{aOR}=1.75,95 \% \mathrm{CI}$ : 1.08-2.83). In the front-line workers group, workers with an educational level of college/university and above $(\mathrm{aOR}=1.39,95 \% \mathrm{CI}: 1.07-1.81)$, a 3-shift/4-team work schedule (aOR $=1.26,95 \% \mathrm{CI}: 1.05-1.52)$, and respiratory diseases $(\mathrm{aOR}=1.48,95 \% \mathrm{CI}: 1.23-1.78)$ had a higher risk of insomnia. In the maintenance and inspection workers group, workers who had household per capita income $<1500 ¥ /$ month $(\mathrm{aOR}=1.29,95 \% \mathrm{CI}: 1.07-1.55)$ or drank alcohol daily had a higher risk of insomnia. 
Table 1. Baseline characteristics of the participants by job category in the study on the prevalence of insomnia and risk factors among 5834 steel workers in Tangshan, China

\begin{tabular}{|c|c|c|c|c|c|c|}
\hline \multirow[b]{2}{*}{ Variable } & \multicolumn{4}{|c|}{$\begin{array}{c}\text { Participants } \\
(\mathrm{N}=5834) \\
{[\mathrm{n}]}\end{array}$} & \multirow[b]{2}{*}{$\chi^{2}$} & \multirow[b]{2}{*}{$\mathrm{p}$} \\
\hline & $\begin{array}{l}\text { front-line } \\
\text { workers } \\
(\mathrm{N}=2848)\end{array}$ & $\begin{array}{c}\text { maintenance } \\
\text { and inspection } \\
\text { workers } \\
(\mathrm{N}=2299)\end{array}$ & $\begin{array}{c}\text { other auxiliary } \\
\text { workers } \\
(\mathrm{N}=687)\end{array}$ & total & & \\
\hline Sex & & & & & 40.931 & $<0.001$ \\
\hline male & 2668 & 2046 & 610 & 5324 & & \\
\hline female & 180 & 253 & 77 & 510 & & \\
\hline Age & & & & & 310.804 & $<0.001$ \\
\hline$<30$ years & 293 & 180 & 19 & 492 & & \\
\hline $30-39$ years & 1030 & 501 & 100 & 1631 & & \\
\hline 40-49 years & 998 & 916 & 327 & 2241 & & \\
\hline$\geq 50$ years & 527 & 702 & 241 & 1470 & & \\
\hline Educational level & & & & & 211.652 & $<0.001$ \\
\hline junior high school or primary & 599 & 482 & 270 & 1351 & & \\
\hline high school & 1369 & 1322 & 338 & 3029 & & \\
\hline college/university and above & 880 & 495 & 79 & 1454 & & \\
\hline Marital status & & & & & 35.419 & $<0.001$ \\
\hline unmarried & 172 & 90 & 11 & 273 & & \\
\hline married & 2602 & 2126 & 661 & 5389 & & \\
\hline other & 74 & 83 & 15 & 172 & & \\
\hline Household per capita income & & & & & 23.214 & $<0.001$ \\
\hline$<1500 ¥ /$ month & 891 & 765 & 162 & 1818 & & \\
\hline$\geq 1500 ¥ /$ month & 1957 & 1534 & 525 & 4016 & & \\
\hline Household per capita living area & & & & & 17.560 & $<0.001$ \\
\hline$<20 \mathrm{~m}^{2}$ & 738 & 702 & 218 & 1658 & & \\
\hline$\geq 20 \mathrm{~m}^{2}$ & 2110 & 1597 & 469 & 4176 & & \\
\hline BMI & & & & & 40.311 & $<0.001$ \\
\hline$<18.5$ & 24 & 20 & 11 & 55 & & \\
\hline $18.5-24.9$ & 1150 & 887 & 339 & 2376 & & \\
\hline $25.0-29.9$ & 1360 & 1188 & 279 & 2827 & & \\
\hline$\geq 30$ & 314 & 204 & 58 & 576 & & \\
\hline Smoking status & & & & & 54.213 & $<0.001$ \\
\hline never & 1251 & 1067 & 274 & 2592 & & \\
\hline ever & 90 & 113 & 37 & 240 & & \\
\hline
\end{tabular}


Table 1. Baseline characteristics of the participants by job category in the study on the prevalence of insomnia and risk factors among 5834 steel workers in Tangshan, China - cont.

\begin{tabular}{|c|c|c|c|c|c|c|}
\hline \multirow[b]{2}{*}{ Variable } & \multicolumn{4}{|c|}{$\begin{array}{c}\text { Participants } \\
(\mathrm{N}=5834) \\
{[\mathrm{n}]}\end{array}$} & \multirow[b]{2}{*}{$\chi^{2}$} & \multirow[b]{2}{*}{$\mathrm{p}$} \\
\hline & $\begin{array}{l}\text { front-line } \\
\text { workers } \\
(\mathrm{N}=2848)\end{array}$ & $\begin{array}{l}\text { maintenance } \\
\text { and inspection } \\
\text { workers } \\
(\mathrm{N}=2299)\end{array}$ & $\begin{array}{c}\text { other auxiliary } \\
\text { workers } \\
(\mathrm{N}=687)\end{array}$ & total & & \\
\hline \multicolumn{7}{|l|}{ Smoking status - cont. } \\
\hline 1-20 cigarettes/day & 1279 & 870 & 284 & 2433 & & \\
\hline$>20$ cigarettes/day & 228 & 249 & 92 & 569 & & \\
\hline Alcohol drinking & & & & & 23.358 & 0.001 \\
\hline never & 1772 & 1413 & 395 & 3580 & & \\
\hline ever & 51 & 52 & 15 & 118 & & \\
\hline$<20 \mathrm{~g} /$ day & 516 & 343 & 118 & 977 & & \\
\hline$\geq 20 \mathrm{~g} /$ day & 509 & 491 & 159 & 1159 & & \\
\hline Tea drinking & & & & & 17.335 & $<0.001$ \\
\hline yes & 1665 & 1211 & 387 & 3263 & & \\
\hline no & 1183 & 1088 & 300 & 2571 & & \\
\hline Physical activity & & & & & 25.688 & $<0.001$ \\
\hline light & 152 & 144 & 29 & 325 & & \\
\hline moderate & 590 & 593 & 154 & 1337 & & \\
\hline heavy & 2106 & 1562 & 504 & 4172 & & \\
\hline Employment relationship & & & & & 45.835 & $<0.001$ \\
\hline contract worker & 2528 & 2016 & 664 & 5208 & & \\
\hline other form & 320 & 283 & 23 & 626 & & \\
\hline Shift work & & & & & 1268.333 & $<0.001$ \\
\hline 3 shifts $/ 4$ teams & 2097 & 1066 & 265 & 3428 & & \\
\hline other shift & 43 & 51 & 186 & 280 & & \\
\hline day shift & 708 & 1182 & 236 & 2126 & & \\
\hline Job stress & & & & & 53.962 & $<0.001$ \\
\hline low stress and high support & 685 & 446 & 144 & 1275 & & \\
\hline low stress and low support & 377 & 345 & 71 & 793 & & \\
\hline high stress and high support & 821 & 581 & 236 & 1638 & & \\
\hline high stress and low support & 965 & 927 & 236 & 2128 & & \\
\hline Life events & & & & & 12.384 & 0.015 \\
\hline 0 & 1806 & 1478 & 454 & 3738 & & \\
\hline 1 & 538 & 478 & 140 & 1156 & & \\
\hline$\geq 2$ & 504 & 343 & 93 & 940 & & \\
\hline
\end{tabular}


Table 1. Baseline characteristics of the participants by job category in the study on the prevalence of insomnia and risk factors among 5834 steel workers in Tangshan, China - cont.

\begin{tabular}{|c|c|c|c|c|c|c|}
\hline \multirow[b]{2}{*}{ Variable } & \multicolumn{4}{|c|}{$\begin{array}{c}\text { Participants } \\
(\mathrm{N}=5834) \\
{[\mathrm{n}]}\end{array}$} & \multirow[b]{2}{*}{$\chi^{2}$} & \multirow[b]{2}{*}{$\mathrm{p}$} \\
\hline & $\begin{array}{l}\text { front-line } \\
\text { workers } \\
(\mathrm{N}=2848)\end{array}$ & $\begin{array}{l}\text { maintenance } \\
\text { and inspection } \\
\text { workers } \\
(\mathrm{N}=2299)\end{array}$ & $\begin{array}{c}\text { other auxiliary } \\
\text { workers } \\
(\mathrm{N}=687)\end{array}$ & total & & \\
\hline Present illness & & & & & 54.836 & $<0.001$ \\
\hline health & 2027 & 1544 & 393 & 3964 & & \\
\hline respiratory diseases & 75 & 49 & 20 & 144 & & \\
\hline other diseases & 746 & 706 & 274 & 1726 & & \\
\hline
\end{tabular}

Table 2. Distribution of insomnia in the workers representing 3 job categories with different baseline characteristics in the study on the prevalence of insomnia and risk factors among 5834 steel workers in Tangshan, China

\begin{tabular}{|c|c|c|c|c|c|c|c|c|c|}
\hline \multirow{3}{*}{ Variable } & \multicolumn{7}{|c|}{$\begin{array}{c}\text { Participants } \\
(\mathrm{N}=5834) \\
{[\mathrm{n}]}\end{array}$} & \multirow{3}{*}{$\chi^{2}$} & \multirow{3}{*}{$\mathrm{p}$} \\
\hline & \multicolumn{2}{|c|}{ front-line workers } & \multicolumn{2}{|c|}{$\begin{array}{l}\text { maintenance and } \\
\text { inspection workers }\end{array}$} & \multicolumn{2}{|c|}{ other auxiliary workers } & \multirow{2}{*}{ total } & & \\
\hline & $\begin{array}{c}\text { total } \\
{[\mathrm{n}]}\end{array}$ & $\begin{array}{l}\text { with insomnia } \\
{[\mathrm{n}(\%)]}\end{array}$ & $\begin{array}{c}\text { total } \\
{[\mathrm{n}]}\end{array}$ & $\begin{array}{c}\text { with insomnia } \\
{[\mathrm{n}(\%)]}\end{array}$ & $\begin{array}{c}\text { total } \\
{[\mathrm{n}]}\end{array}$ & $\begin{array}{c}\text { with insomnia } \\
{[\mathrm{n}(\%)]}\end{array}$ & & & \\
\hline Total & 2848 & $1206(42.3)$ & 2299 & $914(39.8)$ & 687 & $329(47.9)$ & 5834 & 14.673 & 0.001 \\
\hline \multicolumn{10}{|l|}{ Sex } \\
\hline male & 2668 & $1137(42.6)$ & 2046 & $807(39.4)$ & 610 & $285(46.7)$ & 5324 & 11.461 & 0.003 \\
\hline female & 180 & $69(38.3)$ & 253 & $107(42.3)$ & 77 & $44(57.1)$ & 510 & 7.925 & 0.019 \\
\hline \multicolumn{10}{|l|}{ Age } \\
\hline$<30$ years & 293 & $118(40.3)$ & 180 & $66(36.7)$ & 19 & $8(42.1)$ & 492 & 0.688 & 0.709 \\
\hline 30-39 years & 1030 & $430(41.7)$ & 501 & $182(36.3)$ & 100 & $47(47.0)$ & 1631 & 6.037 & 0.049 \\
\hline $40-49$ years & 998 & $446(44.7)$ & 916 & 378 (41.3) & 327 & $173(52.9)$ & 2241 & 13.247 & 0.001 \\
\hline$\geq 50$ years & 527 & $212(40.2)$ & 702 & $288(41.0)$ & 241 & $101(41.9)$ & 1470 & 0.204 & 0.903 \\
\hline \multicolumn{10}{|l|}{ Educational level } \\
\hline junior high school or primary & 599 & $241(40.2)$ & 482 & $203(42.1)$ & 270 & $125(46.3)$ & 1351 & 2.806 & 0.246 \\
\hline high school & 1369 & $583(42.6)$ & 1322 & $535(40.5)$ & 338 & $167(49.4)$ & 3029 & 8.833 & 0.012 \\
\hline college/university and above & 880 & $382(43.4)$ & 495 & $176(35.6)$ & 79 & $37(46.8)$ & 1454 & 9.291 & 0.010 \\
\hline \multicolumn{10}{|l|}{ Marital status } \\
\hline unmarried & 172 & $74(43.0)$ & 90 & $36(40.0)$ & 11 & $4(36.4)$ & 273 & 0.359 & 0.836 \\
\hline married & 2602 & $1098(42.2)$ & 2126 & $839(39.5)$ & 661 & $316(47.8)$ & 5389 & 14.741 & 0.001 \\
\hline other & 74 & $34(45.9)$ & 83 & $39(47.0)$ & 15 & $9(60.0)$ & 172 & 1.018 & 0.601 \\
\hline
\end{tabular}


Table 2. Distribution of insomnia in the workers representing 3 job categories with different baseline characteristics in the study on the prevalence of insomnia and risk factors among 5834 steel workers in Tangshan, China - cont.

\begin{tabular}{|c|c|c|c|c|c|c|c|c|c|}
\hline \multirow{3}{*}{ Variable } & \multicolumn{7}{|c|}{$\begin{array}{c}\text { Participants } \\
(\mathrm{N}=5834) \\
{[\mathrm{n}]}\end{array}$} & \multirow{3}{*}{$\chi^{2}$} & \multirow{3}{*}{$\mathrm{p}$} \\
\hline & \multicolumn{2}{|c|}{ front-line workers } & \multicolumn{2}{|c|}{$\begin{array}{l}\text { maintenance and } \\
\text { inspection workers }\end{array}$} & \multicolumn{2}{|c|}{ other auxiliary workers } & \multirow{2}{*}{ total } & & \\
\hline & $\begin{array}{c}\text { total } \\
{[\mathrm{n}]}\end{array}$ & $\begin{array}{c}\text { with insomnia } \\
{[\mathrm{n}(\%)]}\end{array}$ & $\begin{array}{c}\text { total } \\
{[\mathrm{n}]} \\
\end{array}$ & $\begin{array}{c}\text { with insomnia } \\
{[\mathrm{n}(\%)]}\end{array}$ & $\begin{array}{c}\text { total } \\
{[\mathrm{n}]}\end{array}$ & $\begin{array}{c}\text { with insomnia } \\
{[\mathrm{n}(\%)]}\end{array}$ & & & \\
\hline \multicolumn{10}{|c|}{ Household per capita income } \\
\hline$<1500 ¥ /$ month & 891 & $377(42.3)$ & 765 & $342(44.7)$ & 162 & $86(53.1)$ & 1818 & 6.547 & 0.038 \\
\hline$\geq 1500 ¥ /$ month & 1957 & $829(42.4)$ & 1534 & $572(37.3)$ & 525 & $243(46.3)$ & 4016 & 16.300 & $<0.001$ \\
\hline \multicolumn{10}{|c|}{ Household per capita living area } \\
\hline$<20 \mathrm{~m}^{2}$ & 738 & $335(45.4)$ & 702 & $302(43.0)$ & 218 & $111(50.9)$ & 1658 & 4.232 & 0.121 \\
\hline$\geq 20 \mathrm{~m}^{2}$ & 2110 & $871(41.3)$ & 1597 & $612(38.3)$ & 469 & $218(46.5)$ & 4176 & 10.528 & 0.005 \\
\hline \multicolumn{10}{|l|}{ BMI } \\
\hline$<18.5$ & 24 & $8(33.3)$ & 20 & $8(40.0)$ & 11 & $6(54.5)$ & 55 & 1.414 & 0.493 \\
\hline $18.5-24.9$ & 1150 & $504(43.8)$ & 887 & $361(40.7)$ & 339 & $169(49.9)$ & 2374 & 8.446 & 0.015 \\
\hline $25.0-29.9$ & 1360 & $558(41.0)$ & 1188 & $470(39.6)$ & 279 & $127(45.5)$ & 2259 & 3.351 & 0.187 \\
\hline$\geq 30$ & 314 & $136(43.3)$ & 204 & $75(36.8)$ & 58 & $27(46.6)$ & 576 & 2.915 & 0.233 \\
\hline \multicolumn{10}{|l|}{ Smoking status } \\
\hline never & 1251 & $527(42.1)$ & 1067 & $420(39.4)$ & 274 & $132(48.2)$ & 2592 & 7.215 & 0.027 \\
\hline ever & 90 & $41(45.6)$ & 113 & $54(47.8)$ & 37 & $15(40.5)$ & 240 & 0.594 & 0.743 \\
\hline 1-20 cigarettes/day & 1279 & $525(41.0)$ & 870 & $329(37.8)$ & 284 & $134(47.2)$ & 2433 & 8.005 & 0.018 \\
\hline$>20$ cigarettes/day & 228 & $113(49.6)$ & 249 & $111(44.6)$ & 92 & $48(52.2)$ & 569 & 2.025 & 0.363 \\
\hline \multicolumn{10}{|l|}{ Alcohol drinking } \\
\hline never & 1772 & $735(41.5)$ & 1413 & $520(36.8)$ & 395 & $192(48.6)$ & 3580 & 19.504 & 0.000 \\
\hline ever & 51 & $26(51.0)$ & 52 & $21(40.4)$ & 15 & $6(40.0)$ & 118 & 1.336 & 0.513 \\
\hline$<20$ g/day & 516 & $206(39.9)$ & 343 & $147(42.9)$ & 118 & $53(44.9)$ & 977 & 1.354 & 0.508 \\
\hline$\geq 20 \mathrm{~g} /$ day & 509 & $239(47.0)$ & 491 & $2226(46.0)$ & 159 & $78(49.1)$ & 1159 & 0.446 & 0.800 \\
\hline \multicolumn{10}{|l|}{ Tea drinking } \\
\hline yes & 1665 & $710(42.6)$ & 1211 & $476(39.3)$ & 387 & $180(46.5)$ & 3263 & 7.104 & 0.029 \\
\hline no & 1183 & $496(41.9)$ & 1088 & $438(40.3)$ & 300 & $149(49.7)$ & 2571 & 8.574 & 0.014 \\
\hline \multicolumn{10}{|l|}{ Physical activity } \\
\hline light & 152 & $57(37.5)$ & 144 & $58(40.3)$ & 29 & $18(62.1)$ & 325 & 6.125 & 0.047 \\
\hline moderate & 590 & $228(38.6)$ & 593 & $230(38.8)$ & 154 & $67(43.5)$ & 1337 & 1.314 & 0.518 \\
\hline heavy & 2106 & $921(43.7)$ & 1562 & $626(40.1)$ & 504 & $244(48.4)$ & 4172 & 11.927 & 0.003 \\
\hline \multicolumn{10}{|l|}{ Employment relationship } \\
\hline contract worker & 2528 & $1081(42.8)$ & 2016 & $815(40.4)$ & 664 & $319(48.0)$ & 5208 & 11.959 & 0.003 \\
\hline other form & 320 & $125(39.1)$ & 283 & $99(35.0)$ & 23 & $10(43.5)$ & 626 & 1.447 & 0.485 \\
\hline
\end{tabular}


Table 2. Distribution of insomnia in the workers representing 3 job categories with different baseline characteristics in the study on the prevalence of insomnia and risk factors among 5834 steel workers in Tangshan, China - cont.

\begin{tabular}{|c|c|c|c|c|c|c|c|c|c|}
\hline \multirow{3}{*}{ Variable } & \multicolumn{7}{|c|}{$\begin{array}{c}\text { Participants } \\
(\mathrm{N}=5834) \\
{[\mathrm{n}]}\end{array}$} & \multirow{3}{*}{$\chi^{2}$} & \multirow{3}{*}{$\mathrm{p}$} \\
\hline & \multicolumn{2}{|c|}{ front-line workers } & \multicolumn{2}{|c|}{$\begin{array}{l}\text { maintenance and } \\
\text { inspection workers }\end{array}$} & \multicolumn{2}{|c|}{ other auxiliary workers } & \multirow{2}{*}{ total } & & \\
\hline & $\begin{array}{c}\text { total } \\
{[\mathrm{n}]}\end{array}$ & $\begin{array}{l}\text { with insomnia } \\
{[\mathrm{n}(\%)]}\end{array}$ & $\begin{array}{c}\text { total } \\
{[\mathrm{n}]}\end{array}$ & $\begin{array}{l}\text { with insomnia } \\
{[\mathrm{n}(\%)]}\end{array}$ & $\begin{array}{c}\text { total } \\
{[\mathrm{n}]}\end{array}$ & $\begin{array}{c}\text { with insomnia } \\
{[\mathrm{n}(\%)]}\end{array}$ & & & \\
\hline \multicolumn{10}{|l|}{ Shift work } \\
\hline 3 shifts $/ 4$ teams & 2097 & $917(43.7)$ & 1066 & $430(40.3)$ & 265 & $137(51.7)$ & 3428 & 11.581 & 0.003 \\
\hline other shift & 43 & $17(39.5)$ & 51 & $27(52.9)$ & 186 & $87(46.8)$ & 280 & 1.684 & 0.431 \\
\hline day shift & 708 & $272(38.4)$ & 1182 & $457(38.7)$ & 236 & $105(44.5)$ & 2126 & 3.096 & 0.213 \\
\hline \multicolumn{10}{|l|}{ Job stress } \\
\hline low stress and high support & 685 & $225(32.8)$ & 446 & $146(32.7)$ & 144 & $49(34.0)$ & 1275 & 0.088 & 0.957 \\
\hline low stress and low support & 377 & $162(43.0)$ & 345 & $145(42.0)$ & 71 & $38(53.5)$ & 793 & 3.248 & 0.197 \\
\hline high stress and high support & 821 & $301(36.7)$ & 581 & $209(36.0)$ & 236 & $116(49.2)$ & 1638 & 14.032 & 0.001 \\
\hline high stress and low support & 965 & $518(53.7)$ & 927 & $414(44.7)$ & 236 & $126(53.4)$ & 2128 & 16.814 & $<0.001$ \\
\hline \multicolumn{10}{|l|}{ Life events } \\
\hline 0 & 1806 & $701(38.8)$ & 1478 & $568(38.4)$ & 454 & $205(45.2)$ & 3738 & 7.133 & 0.028 \\
\hline 1 & 538 & $241(44.8)$ & 478 & $192(40.2)$ & 140 & $70(50.0)$ & 1156 & 4.934 & 0.085 \\
\hline$\geq 2$ & 504 & $264(52.4)$ & 343 & $154(44.9)$ & 93 & $54(58.1)$ & 940 & 7.117 & 0.028 \\
\hline \multicolumn{10}{|l|}{ Present illness } \\
\hline health & 2027 & $800(39.5)$ & 1544 & $568(36.8)$ & 393 & $186(43.7)$ & 3964 & 14.725 & 0.001 \\
\hline respiratory diseases & 75 & $40(53.3)$ & 49 & $27(55.1)$ & 20 & $13(65.0)$ & 144 & 0.877 & 0.645 \\
\hline other diseases & 746 & $366(49.1)$ & 706 & $319(45.2)$ & 274 & $130(47.4)$ & 1726 & 2.195 & 0.334 \\
\hline
\end{tabular}

\section{DISCUSSION}

As shown in this study, the prevalence of insomnia was $42.0 \%$ in the total steel workers in China. Compared with the prevalence reported in most previous works, the insomnia prevalence revealed in this study is much higher. The prevalence was reported range 11.9-37.75\% among the general population, community residents, adults, and the elderly in China [6,7]. Meanwhile, it was also higher than $24 \%$ reported for U.S. workers [9], 32.9\% for Korean workers [10], and $4.7 \%$ for male workers in Japan [29], but similar to $39.7 \%$ for gas transmission industry workers in Iran [30].
The steel workers in the other auxiliary workers group had the highest rate of insomnia (47.9\%), while this rate was the lowest in the maintenance and inspection workers group (39.8\%). The following potential reasons for this striking discrepancy could be considered and proven in future studies:

1. The work environment (occupational hazards, hazardous circumstances, etc.) and work organization form (working time, job stability, workload, etc.) are very different, because they take on different tasks.

2. Some personal characteristics, such as personality, psychosocial stress, way of live, behavioral habits, etc., should be taken into account. 


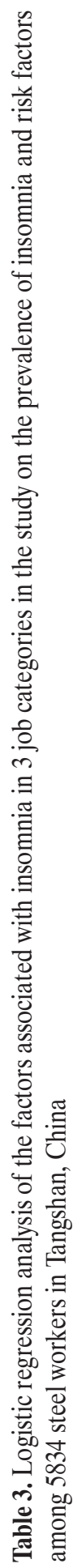

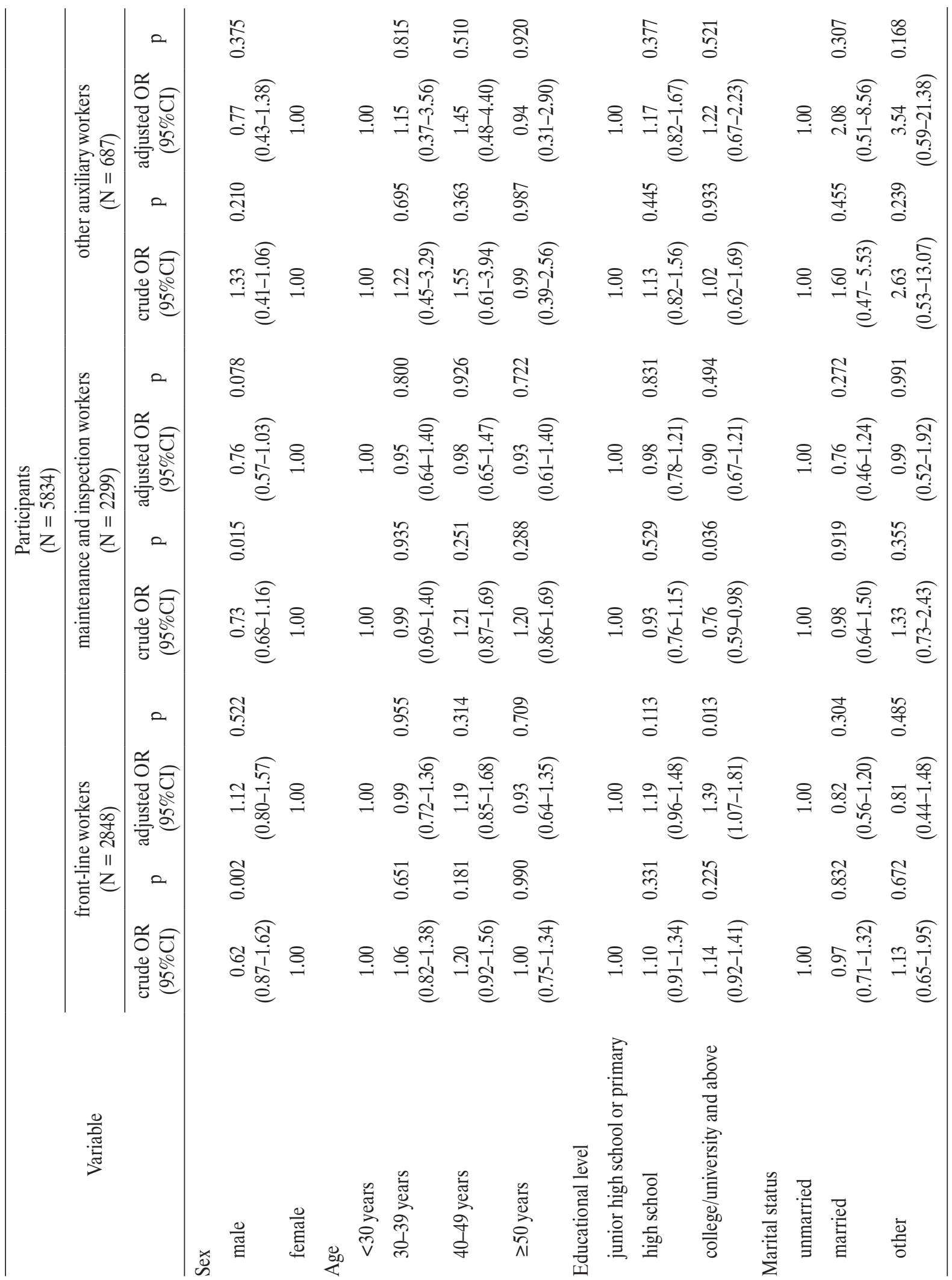




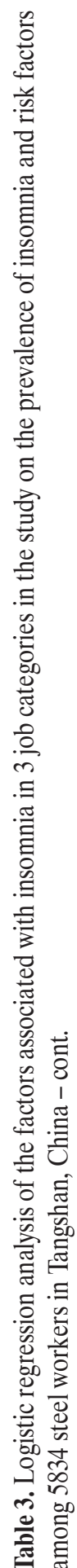

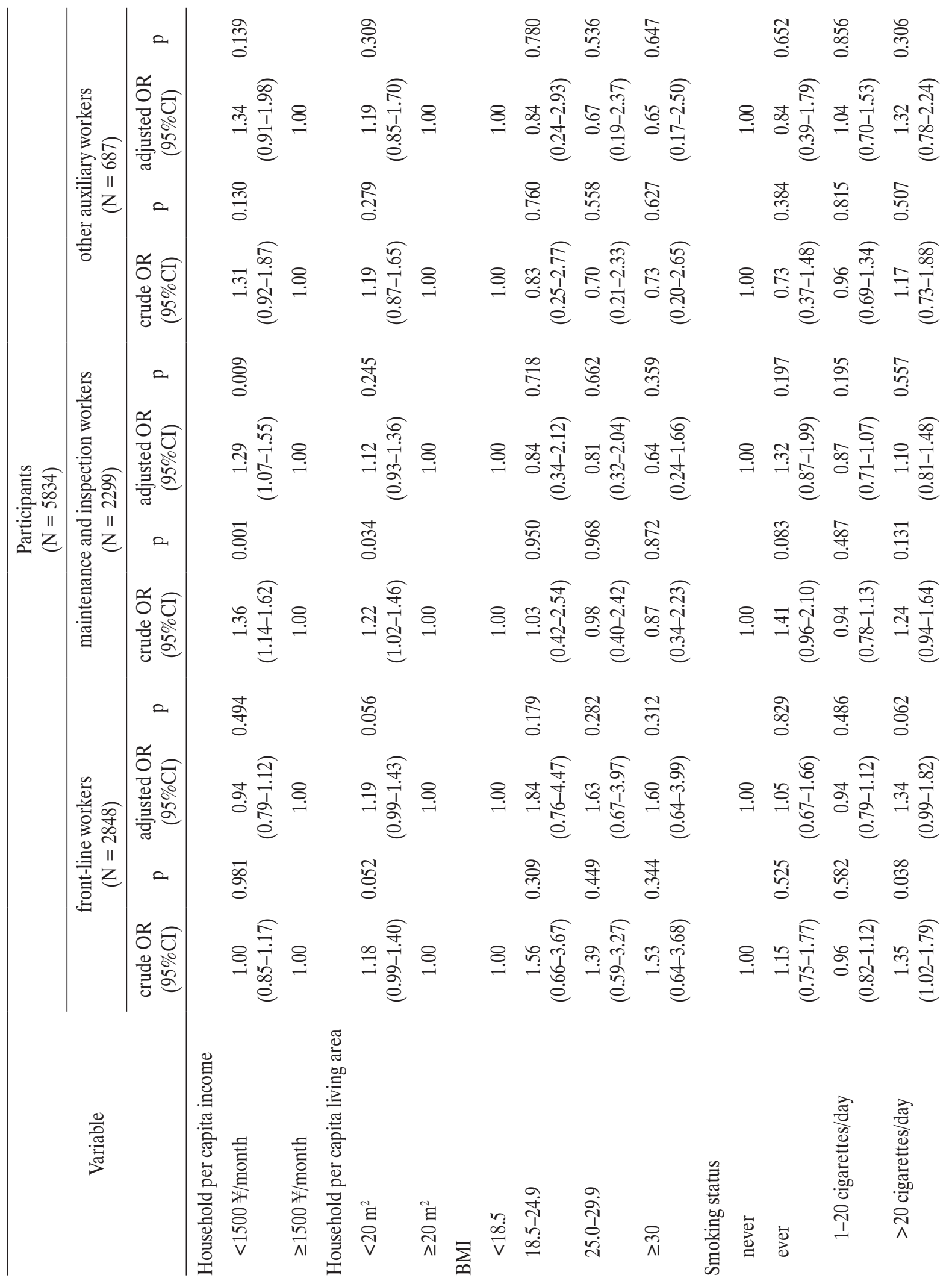




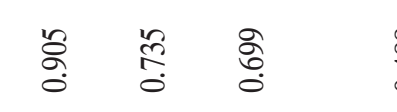

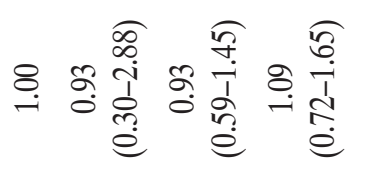

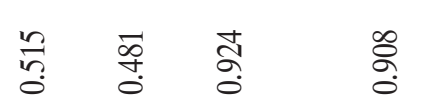

8.

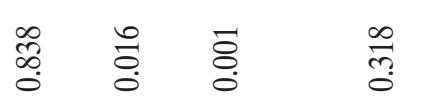

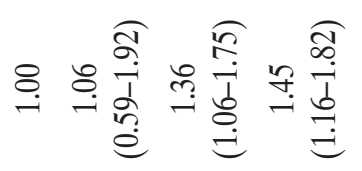

愈 乫

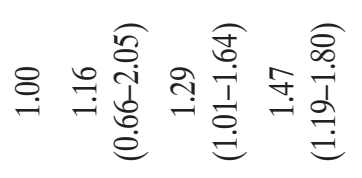

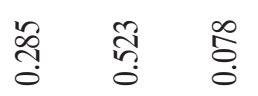

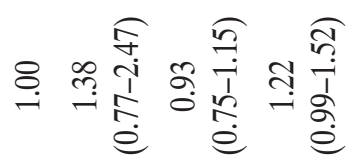

言 형

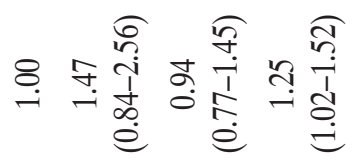

梁

赵孛

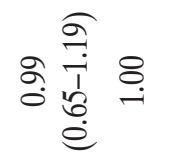

总

\%

施

总

章

\%

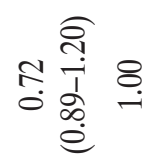

范

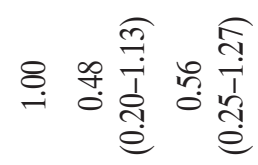

응

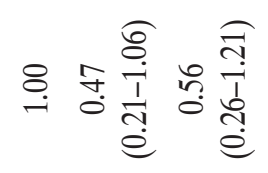

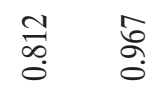

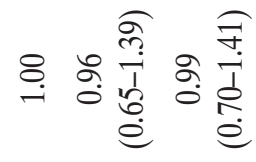

尔

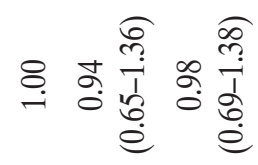

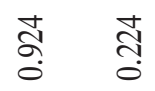

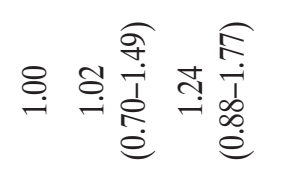

莬

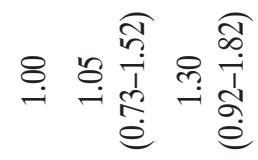

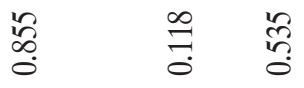

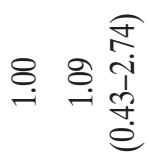

器

\&.

悉

\&

:

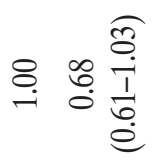

$\bar{g}$

억

\&

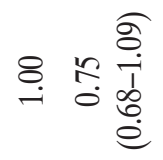

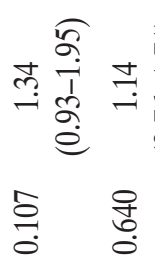

尔 气ิ

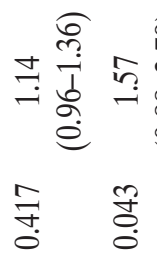

공 :

营

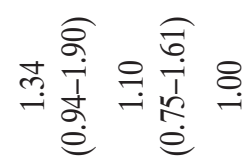

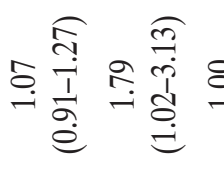

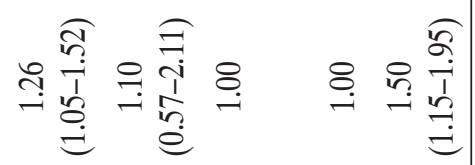

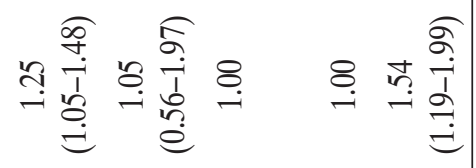

ஓ्ठ

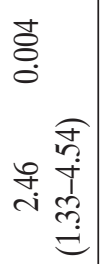

웅

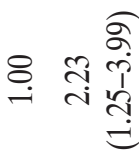

$\stackrel{\infty}{\overparen{0}}$

\&

용

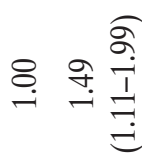

$\stackrel{8}{8}$

奇

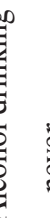

喜喜量

量

竞

謩产

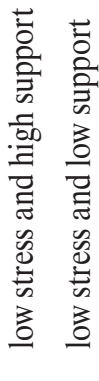




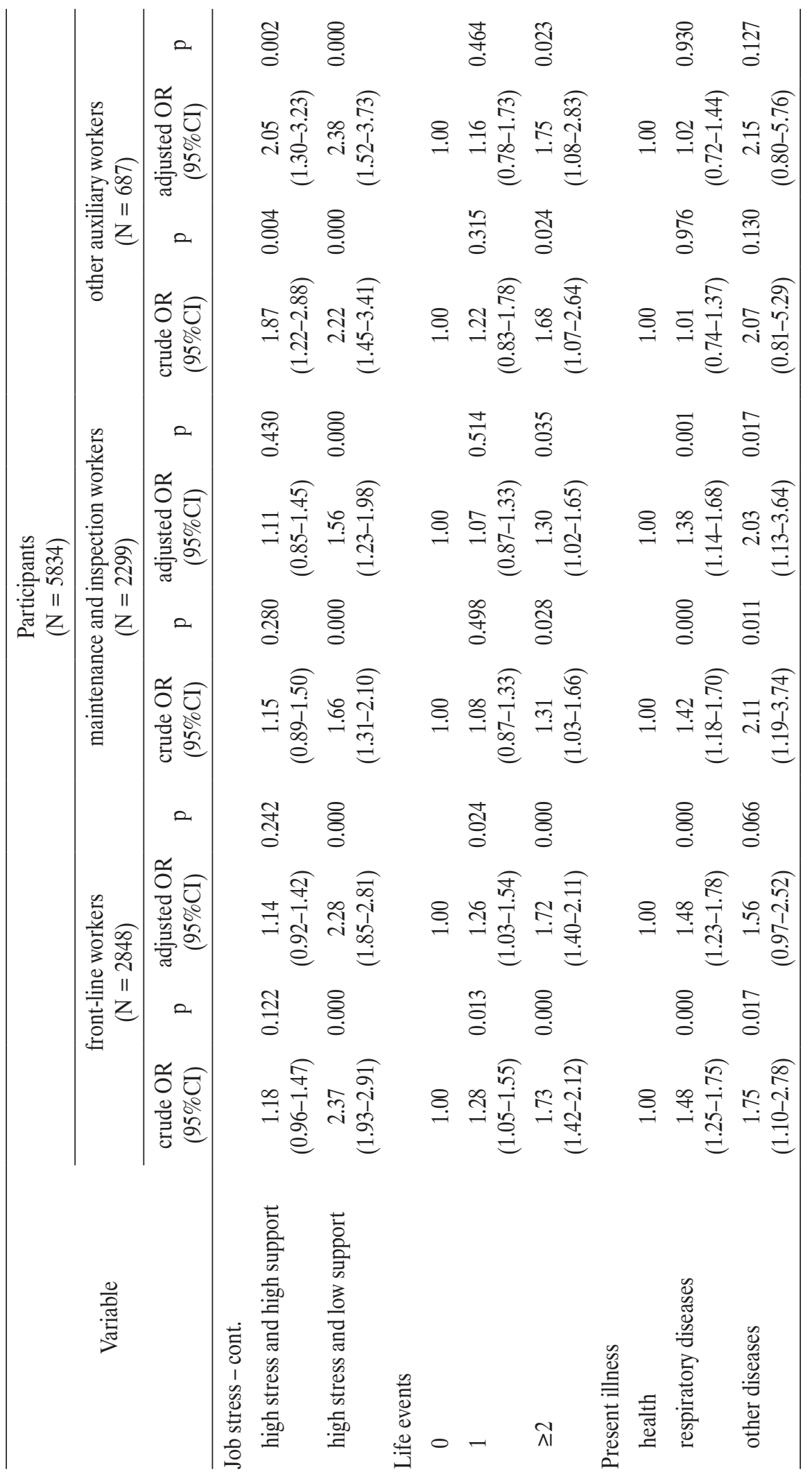


Table 4. Logistic regression analysis of the factors associated with insomnia in steel workers in the study on the prevalence of insomnia and risk factors among 5834 steel workers in Tangshan, China

\begin{tabular}{|c|c|c|c|c|}
\hline \multirow[t]{2}{*}{ Variable } & \multicolumn{4}{|c|}{$\begin{array}{l}\text { Participants } \\
(\mathrm{N}=5834)\end{array}$} \\
\hline & crude OR $(95 \% \mathrm{CI})$ & $\mathrm{p}$ & adjusted OR $(95 \% \mathrm{CI})$ & $\mathrm{p}$ \\
\hline \multicolumn{5}{|l|}{ Sex } \\
\hline male & $0.95(0.79-1.14)$ & 0.579 & $0.87(0.71-1.07)$ & 0.196 \\
\hline female & 1.00 & & 1.00 & \\
\hline \multicolumn{5}{|l|}{ Age } \\
\hline$<30$ years & 1.00 & & 1.00 & \\
\hline $30-39$ years & $1.51(0.86-1.30)$ & 0.584 & $0.99(0.78-1.26)$ & 0.942 \\
\hline 40-49 years & $1.25(1.03-1.53)$ & 0.027 & $1.13(0.88-1.45)$ & 0.358 \\
\hline$\geq 50$ years & $1.08(0.88-1.33)$ & 0.467 & $0.93(0.71-1.21)$ & 0.571 \\
\hline \multicolumn{5}{|l|}{ Educational level } \\
\hline junior high school or primary & 1.00 & & 1.00 & \\
\hline high school & $1.01(0.89-1.15)$ & 0.850 & $1.10(0.96-1.26)$ & 0.185 \\
\hline college/university and above & $0.95(0.82-1.11)$ & 0.521 & $1.16(0.97-1.39)$ & 0.106 \\
\hline \multicolumn{5}{|l|}{ Marital status } \\
\hline unmarried & 1.00 & & 1.00 & \\
\hline married & $1.00(0.78-1.28)$ & 0.987 & $0.82(0.61-1.09)$ & 0.170 \\
\hline other & $1.27(0.87-1.87)$ & 0.221 & $0.94(0.62-1.43)$ & 0.766 \\
\hline \multicolumn{5}{|l|}{ Household per capita income } \\
\hline$<1500 ¥ /$ month & $1.15(1.03-1.28)$ & 0.017 & $1.11(0.98-1.25)$ & 0.090 \\
\hline$\geq 1500 ¥ /$ month & 1.00 & & 1.00 & \\
\hline \multicolumn{5}{|l|}{ Household per capita living area } \\
\hline$<20 \mathrm{~m}^{2}$ & $1.20(1.07-1.34)$ & 0.002 & $1.16(1.03-1.31)$ & 0.002 \\
\hline$\geq 20 \mathrm{~m}^{2}$ & 1.00 & & 1.00 & \\
\hline \multicolumn{5}{|l|}{ BMI } \\
\hline$<18.5$ & 1.00 & & 1.00 & \\
\hline $18.5-24.9$ & $1.16(0.67-1.99)$ & 0.603 & $1.15(0.66-2.01)$ & 0.632 \\
\hline $25.0-29.9$ & $1.04(0.60-1.79)$ & 0.898 & $1.05(0.60-1.84)$ & 0.864 \\
\hline$\geq 30$ & $1.06(0.60-1.86)$ & 0.849 & $0.95(0.54-1.71)$ & 0.885 \\
\hline \multicolumn{5}{|l|}{ Smoking status } \\
\hline never & 1.00 & & 1.00 & \\
\hline ever & $1.19(0.91-1.55)$ & 0.207 & $1.12(0.85-1.49)$ & 0.414 \\
\hline 1-20 cigarettes/day & $0.96(0.86-1.07)$ & 0.463 & $0.93(0.82-1.05)$ & 0.245 \\
\hline$>20$ cigarettes/day & $1.28(1.07-1.54)$ & 0.007 & $1.22(0.99-1.48)$ & 0.053 \\
\hline
\end{tabular}


Table 4. Logistic regression analysis of the factors associated with insomnia in steel workers in the study on the prevalence of insomnia and risk factors among 5834 steel workers in Tangshan, China - cont.

\begin{tabular}{|c|c|c|c|c|}
\hline \multirow[t]{2}{*}{ Variable } & \multicolumn{4}{|c|}{$\begin{array}{c}\text { Participants } \\
(\mathrm{N}=5834)\end{array}$} \\
\hline & crude OR $(95 \% \mathrm{CI})$ & $\mathrm{p}$ & adjusted OR $(95 \% \mathrm{CI})$ & $\mathrm{p}$ \\
\hline \multicolumn{5}{|l|}{ Alcohol drinking } \\
\hline never & 1.00 & & 1.00 & \\
\hline ever & $1.20(0.83-1.74)$ & 0.328 & $1.16(0.79-1.70)$ & 0.461 \\
\hline$<20$ g/day & $1.05(0.91-1.21)$ & 0.521 & $1.07(0.92-1.25)$ & 0.355 \\
\hline$\geq 20 \mathrm{~g} /$ day & $1.30(1.14-1.49)$ & 0.000 & $1.29(1.11-1.49)$ & 0.001 \\
\hline \multicolumn{5}{|l|}{ Tea drinking } \\
\hline yes & $0.73(0.89-1.10)$ & 0.000 & $0.71(0.59-0.86)$ & 0.001 \\
\hline no & 1.00 & & 1.00 & \\
\hline \multicolumn{5}{|l|}{ Physical activity } \\
\hline light & 1.00 & & 1.00 & \\
\hline moderate & $0.93(0.73-1.20)$ & 0.584 & $0.92(0.72-1.19)$ & 0.539 \\
\hline heavy & $1.09(0.86-1.37)$ & 0.482 & $1.06(0.83-1.34)$ & 0.657 \\
\hline \multicolumn{5}{|l|}{ Employment form } \\
\hline contract worker & 1.00 & & 1.00 & \\
\hline other form & $0.74(0.68-0.96)$ & 0.000 & $0.92(0.76-1.12)$ & 0.923 \\
\hline \multicolumn{5}{|l|}{ Shift work } \\
\hline 3 shifts $/ 4$ teams & $1.18(1.06-1.32)$ & 0.003 & $1.21(1.08-1.37)$ & 0.001 \\
\hline other shift & $1.36(1.06-1.75)$ & 0.016 & $1.17(0.89-1.54)$ & 0.265 \\
\hline day shift & 1.00 & & 1.00 & \\
\hline \multicolumn{5}{|l|}{ Job stress } \\
\hline low stress and high support & 1.00 & & 1.00 & \\
\hline low stress and low support & $1.57(1.31-1.88)$ & 0.000 & $1.53(1.27-1.84)$ & 0.001 \\
\hline high stress and high support & $1.26(1.08-1.47)$ & 0.003 & $1.22(1.04-1.43)$ & 0.012 \\
\hline high stress and low support & $2.01(1.74-2.33)$ & 0.000 & $1.96(1.69-2.28)$ & 0.000 \\
\hline \multicolumn{5}{|l|}{ Life events } \\
\hline 0 & 1.00 & & 1.00 & \\
\hline 1 & $1.18(1.04-1.35)$ & 0.014 & $1.18(1.03-1.36)$ & 0.016 \\
\hline$\geq 2$ & $1.55(1.34-1.79) 0.000$ & & $1.55(1.69-2.28)$ & 0.000 \\
\hline \multicolumn{5}{|l|}{ Present illness } \\
\hline health & 1.00 & & 1.00 & \\
\hline respiratory diseases & $1.39(1.24-1.56)$ & 0.000 & $1.36(1.20-1.54)$ & 0.000 \\
\hline other diseases & $1.94(1.39-2.71)$ & 0.000 & $1.79(1.27-2.52)$ & 0.001 \\
\hline
\end{tabular}


The 3 groups of workers under analysis exhibit significant differences in terms of socio-demographic characteristics, living and behavioral habits, job characteristics, and personal characteristics considered in this study. Therefore, the authors analyzed the relationship between each variable and insomnia while adjusting for the other remaining variables using a multivariate logistic regression analysis. The results of the multivariate logistic regression analysis show that job stress and life events are common risk factors of insomnia for workers in all 3 job categories. The authors also obtained the same results in all the participants, when analyzing the influencing factors of insomnia (Table 4). Meanwhile, they also observed that alcohol consumption in the quantity of $>20 \mathrm{~g} /$ day, shift work and current illness were associated with insomnia in all the participants.

Job stress is another risk factor of insomnia, which has been reported in many previous studies [18]. The results presented in this study found that steel workers with high stress and low social support were most likely to suffer from insomnia, and that steel workers struggling with stress had a higher risk of insomnia than steel workers without stress. This finding proves that high job stress is closely associated with an increased risk of insomnia in steel workers in China, just like in workers in other industries or companies. The authors recommend that, in order to ensure a better health condition of steel workers, they should undergo stress management training and team building activities to improve their stress-coping strategies and sleep quality.

The authors also found that the 3 groups of steel workers who had experienced more life events in the past 12 months were associated with a greater risk of insomnia. A study of stressful life events, insomnia, and suicide risks in Chinese adolescents has reached the same hypothesis that stressful life events are related to insomnia [31]. The same results have also been reported in studies that focused on U.S. Army soldiers prior to deployment [32], and on a community of older people in 4 cities in the Hebei Province,
China [33]. These results suggest that, when conducting occupational health management, more attention should be paid to the workers who experience major life events, and that necessary help and mental health promotion should be provided to them. However, the effects of positive and negative events associated with insomnia have not been analyzed, which could be the subject of future studies. There were several limitations associated with this study. First, this was a cross-sectional study, so it was impossible to explore the causal effect between insomnia and numerous factors. Second, the participants came from a steel company located in a heavily industrialized city in northern China, so the results of this study are limited in terms of generalization to all Chinese steel workers. Third, work conditions and the workplace environment were not evaluated, which could exclude some confounding factors and influence others. Finally, the mental health of the participants should be thoroughly evaluated in order to analyze the insomnia status of steel workers in China.

\section{CONCLUSIONS}

This study suggests that the prevalence of insomnia was different in the steel workers groups representing 3 job categories, and that its rate was the highest among other auxiliary workers. Job stress and life events are the common risk factors of insomnia in the 3 job categories of steel workers under analysis. When preparing the appropriate strategies for the prevention and treatment of insomnia among steel workers, policy-makers are recommended to pay more attention to job stress. Furthermore, psychological counseling should be provided to workers who have experienced recent life events.

\section{ACKNOWLEDGMENTS}

The authors would like to express their great appreciation to the collaborating agency - the Tangshan Hongci Hospital. They would also like to thank all the teachers and students who took part in the research design and field investigations. 


\section{REFERENCES}

1. Roth T, Coulouvrat C, Hajak G, Lakoma MD, Sampson NA, Shahly V, et al. Prevalence and perceived health associated with insomnia based on DSM-IV-TR; International Statistical Classification of Diseases and Related Health Problems, Tenth Revision; and Research Diagnostic Criteria/International Classification of Sleep Disorders, Second Edition criteria: results from the America Insomnia Survey. Biol Psychiatry. 2011;69(6):592600, https://doi.org/10.1016/j.biopsych.2010.10.023.

2. Uhlig BL, Sand T, Odegard SS, Hagen K. Prevalence and associated factors of DSM-V insomnia in Norway: the Nord-Trondelag Health Study (HUNT 3). Sleep Med. 2014;15(6):70813, https://doi.org/10.1016/j.sleep.2014.01.018.

3. Li RH, Wing YK, Ho SC, Fong SY. Gender differences in insomnia - a study in the Hong Kong Chinese population. J Psychosom Res. 2002;53(1):601-9.

4. Soldatos CR, Allaert FA, Ohta T, Dikeos DG. How do individuals sleep around the world? Results from a single-day survey in ten countries. Sleep Med. 2005;6(1):5-13, https:// doi.org/10.1016/j.sleep.2004.10.006.

5. Li MR, Lai HL. Insomnia and its associated factors in incarcerated adults: A cross-sectional study. Nurs Health Sci. 2019;21:330-5, https://doi.org/10.1111/nhs.12600.

6. Zou Y, Chen Y, Yu W, Chen T, Tian Q, Tu Q, et al. The prevalence and clinical risk factors of insomnia in the Chinese elderly based on comprehensive geriatric assessment in Chongqing population. Psychogeriatrics. 2019;19(4):384-90, https://doi.org/10.1111/psyg.12402.

7. Ma Y, Hu Z, Qin X, Chen R, Zhou Y. Prevalence and socioeconomic correlates of insomnia among older people in Anhui, China. Australas J Ageing. 2018;37(3):E91-6, https://doi. org/10.1111/ajag.12531.

8. Li L, Wang YY, Wang SB, Zhang L, Li L, Xu DD, et al. Prevalence of sleep disturbances in Chinese university students: a comprehensive meta-analysis. J Sleep Res. 2018;27(3):e12648, https://doi.org/10.1111/jsr.12648.

9. Kessler RC, Berglund PA, Coulouvrat C, Hajak G, Roth T, Shahly V, et al. Insomnia and the performance of US workers: results from the America insomnia survey. Sleep. 2011;34(9): 1161-71, https://doi.org/10.5665/SLEEP.1230.

10. Kim HC, Kim BK, Min KB, Min JY, Hwang SH, Park SG. Association between job stress and insomnia in Korean workers. J Occup Health. 2011;53(3):164-74, https://doi. org/10.1539/joh.10-0032-oa.

11. Yoshioka E, Saijo Y, Kita T, Satoh H, Kawaharada M, Kishi R. Effect of the interaction between employment level and psychosocial work environment on insomnia in male Japanese public service workers. Int J Behav Med. 2013;20(3):355-64, https://doi.org/10.1007/s12529-012-9230-9.

12. Metlaine A, Sauvet F, Gomez-Merino D, Elbaz M, Delafosse JY, Leger D, et al. Association between insomnia symptoms, job strain and burnout syndrome: a cross-sectional survey of 1300 financial workers. BMJ Open. 2017;7(1):e012816, https://doi.org/10.1136/bmjopen-2016-012816.

13. Lin QF, Xia QQ, Zeng YL, Wu XY, Ye LF, Yao LT, et al. Prevalence of migraine in Han Chinese of Fujian province: An epidemiological study. Medicine (Baltimore). 2018;97(52): e13500, https://doi.org/10.1097/MD.0000000000013500.

14. Troxel WM, Buysse DJ, Matthews KA, Kip KE, Strollo PJ, Hall M, et al. Sleep symptoms predict the development of the metabolic syndrome. Sleep. 2010;33(12):1633-40, https://doi.org/10.1093/sleep/33.12.1633.

15. Ford ES, Cunningham TJ, Giles WH, Croft JB. Trends in insomnia and excessive daytime sleepiness among U.S. adults from 2002 to 2012. Sleep Med. 2015;16(3):372-8, https://doi.org/10.1016/j.sleep.2014.12.008.

16. Walsh JK, Engelhardt CL. The direct economic costs of insomnia in the United States for 1995. Sleep. 1999;22 Suppl 2:S386-93.

17. Chatterjee K, Ambekar P. Study of insomnia in rotating shift-workers. Ind Psychiatry J. 2017;26(1):82-5, https://doi. org/10.4103/ipj.ipj_59_17.

18. Yang B, Wang Y, Cui F, Huang T, Sheng P, Shi T, et al. Association between insomnia and job stress: a meta-analysis. Sleep Breath. 2018;22(4):1221-31, https://doi.org/10.1007/ s11325-018-1682-y. 
19. Lim HM, Kang W, Park WJ, Jang KH, Ann JS, Moon JD. Insomnia and hearing impairment among occupational noise exposed male workers. Ann Occup Environ Med. 2017;29:36, https://doi.org/10.1186/s40557-017-0195-7.

20. Guo Y, Hu H, Liu Y, Leng Y, Gao X, Cui Q, et al. Gender differences in the relationship between alcohol consumption and insomnia in the northern Chinese population. PLoS One. 2018;13(12):e0207392, https://doi.org/10.1371/journal. pone.0207392.

21. Vallieres A, Azaiez A, Moreau V, LeBlanc M, Morin CM. Insomnia in shift work. Sleep Med. 2014;15(12):1440-8, https://doi.org/10.1016/j.sleep.2014.06.021.

22. Qu NN, Li KJ. [Study on the reliability and validity of international physical activity questionnaire (Chinese Vision, IPAQ)]. Zhonghua Liu Xing Bing Xue Za Zhi. 2004;25(3): 265-8. Chinese.

23. Ren YJ, Su M, Liu QM, Tan YY, Du YK, Li LM, et al. Validation of the Simplified Chinese-character Version of the International Physical Activity Questionnaire-Long Form in Urban Community-dwelling Adults: a Cross-sectional Study in Hangzhou, China. Biomed Environ Sci. 2017;30(4):25563, https://doi.org/10.3967/bes2017.035.

24. Costa G. Shift work and occupational medicine: an overview. Occup Med (Lond). 2003;53(2):83-8, https://doi.org/ 10.1093/occmed/kqg045.

25. Karasek R, Brisson C, Kawakami N, Houtman I, Bongers P, Amick B. The Job Content Questionnaire (JCQ): an instrument for internationally comparative assessments of psychosocial job characteristics. J Occup Health Psychol. 1998;3(4):322-55.

26. Dai JM, Collins S, Yu HZ, Fu H. Combining job stress models in predicting burnout by hierarchical multiple regressions: a cross-sectional investigation in Shanghai. J Occup
Environ Med. 2008;50(7):785-90, https://doi.org/10.1097/ JOM.0b013e318167750a.

27. Han X, Yang Y, Chen Y, Gao L, Yin X, Li H, et al. Association between insomnia and atrial fibrillation in a Chinese population: A cross-sectional study. Clin Cardiol. 2017;40(9):765-9, https://doi.org/10.1002/clc.22731.

28. Jeong HS, Jeon Y, Ma J, Choi Y, Ban S, Lee S, et al. Validation of the Athens Insomnia Scale for screening insomnia in South Korean firefighters and rescue workers. Qual Life Res. 2015;24(10):2391-5, https://doi.org/10.1007/s11136015-0986-7.

29. Nomura K, Nakao M, Takeuchi T, Yano E. Associations of insomnia with job strain, control, and support among male Japanese workers. Sleep Med. 2009;10(6):626-9, https://doi. org/10.1016/j.sleep.2008.06.010.

30. Moradifar R, Hoveidi H, Givehchi S, Talebi F. Examining fatigue and insomnia symptoms among workers of a gas transmission industry in 2013. Electron Physician. 2014;6(2):82731, https://doi.org/10.14661/2014.932-827-831.

31. Liu BP, Wang XT, Liu ZZ, Wang ZY, Liu X, Jia CX. Stressful life events, insomnia and suicidality in a large sample of Chinese adolescents. J Affect Disord. 2019;249:404-9, https://doi.org/10.1016/j.jad.2019.02.047.

32. Taylor DJ, Pruiksma KE, Hale WJ, Kelly K, Maurer D, Peterson AL, et al. Prevalence, Correlates, and Predictors of Insomnia in the US Army prior to Deployment. Sleep. 2016;39(10):1795-806, https://doi.org/10.5665/sleep. 6156.

33. Wang YM, Chen HG, Song M, Xu SJ, Yu LL, Wang L, et al. Prevalence of insomnia and its risk factors in older individuals: a community-based study in four cities of Hebei Province, China. Sleep Med. 2016;19:116-22, https://doi.org/ 10.1016/j.sleep.2015.10.018.

This work is available in Open Access model and licensed under a Creative Commons Attribution-NonCommercial 3.0 Poland License - http://creativecommons.org/ licenses/by-nc/3.0/pl/deed.en. 